\title{
Lateral Pelvic Node Metastasis in Locally Advanced Rectal Cancer: Are We Exaggerating or Ignoring?
}

\author{
Gyu-Seog Choi, MD, PhD (D) \\ Colorectal Cancer Center, Kyungpook National University Chilgok Hospital, School of Medicine, Kyungpook National \\ University, Daegu, Korea
}

\begin{abstract}
Although the importance of the lateral pelvic node (LPN) in rectal cancer has been described for more than a century, treatments remain in parallel with surgical resection in the East, especially in Japan, whereas neoadjuvant concurrent chemoradiation (nCCRT) is preferred in the West.
\end{abstract}

A recent randomized clinical trial (JCOG0212) in Japan demonstrated the efficacy of LPN dissection (LPND) with total mesorectal excision (TME), which had a lower local recurrence rate $(7 \%)$ compared with TME alone (13\%). ${ }^{1}$ However, this strategy may be hardly accepted by Western surgeons since nCCRT is ignored in most advanced rectal cancers. Moreover, the rate of metastatic LPN was $7.4 \%$ in the LPND group, indicating that the majority of patients underwent potentially unnecessary LPND. On the other hand, there is ample evidence that nCCRT and TME are insufficient to irradicate metastatic LPNs when LPND is ignored; rather, they cause a considerable incidence of local recurrence, especially in the lateral pelvic wall. ${ }^{2-4}$

The study by Hida et al. ${ }^{5}$ in this issue of Annals of Surgical Oncology, through careful review of pelvic magnetic resonance imaging in a large series of Japanese rectal cancer patients, highlighted the prognostic impact of LPND compared with nCCRT alone on improved 5-year estimated overall survival $(81.9 \%$ vs. $67.3 \%)$ and relapsefree survival $(69.4 \%$ vs. $51.6 \%)$ in patients with enlarged LPNs (5-10 mm). In addition, LPND more prominently

(C) Society of Surgical Oncology 2021

First Received: 19 July 2021

Accepted: 24 July 2021;

Published Online: 11 August 2021

G.-S. Choi, MD, PhD

e-mail: kyuschoi@mail.knu.ac.kr enhanced relapse-free survival in the subgroup with neoadjuvant treatment than in the TME-alone group $(75.2 \%$ vs. $33.3 \%)$. These results are similar to those of a previous collaborative pooled analysis by the Lateral Node Study Consortium, ${ }^{2}$ in which most of the prognostic parameters, such as 5-year distant recurrence (13.5\% vs. $30.8 \%$ ), cancer-specific survival (94.1\% vs. $79.4 \%)$, local recurrence $(5.7 \%$ vs $25.7 \%)$, and, in particular, lateral pelvic recurrence $(5.7 \%$ vs. $19.5 \%)$, were significantly better with LPND than with TME alone in rectal cancer patients with enlarged LPNs $(>7 \mathrm{~mm})$, even after nCCRT.

Taking together the lessons from the literature reported on both sides of the world, it is high time to merge these two important treatment modalities in order to provide better clinical outcomes by 'selective' LPND with a combination of nCCRT for patients with highly suspicious metastatic LPNs. Therefore, the selection of high-risk patients with metastatic LPN is of paramount importance. As shown in the study by Hida et al., ${ }^{5}$ a 5-mm short-axis diameter may be a simple cut-off for the indication of LPND, although the shape, consistency, and responsiveness of LPNs to nCCRT were also taken into consideration. Unfortunately, the investigators applied this size criterion to non-treated LPNs in the surgery (TME + LPND) group mixed with post-treated LPNs in the neoadjuvant + surgery group, which might have biased the results.

A practical reason why LPND failed to account for the treatment of suspicious metastatic LPNs in the West may be its unfamiliarity and the fear of a higher rate of complications. Indeed, Hida et al. ${ }^{5}$ showed a significantly longer operation time, a larger amount of blood loss, and a higher rate of grade 2-3 complications in the LPND group $(44.5 \%)$ than in the TME-alone group (33.2\%). However, 
the JCOG0212 trial reported no significant difference in overall complications between the groups (TME vs. TME + LPND) in non-radiated patients. ${ }^{6}$

Regarding the morbidity of LPND, systematic surgical training is essential for safe LPND. The education of young surgeons to delineate fascial planes and other important anatomical landmarks in the deep pelvic cavity is becoming easier, especially in the era of minimally invasive surgery. ${ }^{7}$ By using fluorescence and three-dimensional reconstruction of imaging, several attempts have been made to standardize and improve the quality of LPND based on surgical anatomy. ${ }^{8}$

While this long-standing debate may continue in the near future, it seems clearer that LPND should not be exaggerated for potentially unnecessary patients, nor should the effectiveness of LPND or nCCRT be ignored for patients with LPN involvement. Therefore, further efforts are definitely needed to selectively combine all available treatment modalities to provide better service to patients with advanced rectal cancer.

DISCLOSURE This study has not been performed under any commercial sponsorship or grant.

\section{REFERENCES}

1. Fujita S, Mizusawa J, Kanemitsu Y, et al. Colorectal Cancer Study Group of Japan Clinical Oncology Group. Mesorectal excision with or without lateral lymph node dissection for clinical stage II/ III lower rectal cancer (JCOG0212): A multicenter, randomized controlled, noninferiority trial. Ann Surg. 2017;266:201-7.
2. Ogura A, Konishi T, Cunningham C, et al. Lateral Node Study Consortium. Neoadjuvant (Chemo)radiotherapy with total mesorectal excision only is not sufficient to prevent lateral local recurrence in enlarged nodes: Results of the multicenter lateral node study of patients with low cT3/4 rectal cancer. J Clin Oncol. 2019;37:33-43.

3. Kim TH, Jeong SY, Choi DH, et al. Lateral lymph node metastasis is a major cause of locoregional recurrence in rectal cancer treated with preoperative chemoradiotherapy and curative resection. Ann Surg Oncol. 2008;15:729-37.

4. Kim HJ, Choi GS, Park JS, et al. Optimal treatment strategies for clinically suspicious lateral pelvic lymph node metastasis in rectal cancer. Oncotarget. 2017;8:100724-33.

5. Hida K, Nishzaki D, Sumii A, et al.; Japan Society of Laparoscopic Colorectal Surgery. Prognostic impact of lateral pelvic node dissection on the survival of patients in low rectal cancer subgroups based on lymph node size. Ann Surg Oncol. 2021. https://doi.org/10.1245/s10434-021-10312-7.

6. Fujita S, Akasu T, Mizusawa J, et al. Colorectal Cancer Study Group of Japan Clinical Oncology Group. Postoperative morbidity and mortality after mesorectal excision with and without lateral lymph node dissection for clinical stage II or stage III lower rectal cancer (JCOG0212): results from a multicentre, randomised controlled, non-inferiority trial. Lancet Oncol. 2012;13:616-21.

7. Choi GS, Kim HJ, Park JS, et al. Minimally invasive approach for lateral pelvic node dissection: a standardization based on surgical anatomy. Dis Colon Rectum. 2019;62:1550.

8. Kim HJ, Choi GS, Park JS, et al. S122: impact of fluorescence and $3 \mathrm{D}$ images to completeness of lateral pelvic node dissection. Surg Endosc. 2020;34:469-76.

Publisher's Note Springer Nature remains neutral with regard to jurisdictional claims in published maps and institutional affiliations. 